\title{
Generalized Similarity Judgments: An Alternative Explanation for Choice Anomalies
}

JONATHAN W. LELAND

Department of Social \& Decision Sciences, Carnegie Mellon University

\section{Abstract}

Rubinstein's (1988) procedure for choosing between risky prospects, based, in part, upon similarities between prizes and probabilities across lotteries, is modified and extended to apply to a more general class of binary choices. This modified procedure is shown to imply behaviors following from Loomes and Sugden's (1982) Regret Theory, although under more general conditions, and provides an alternative explanation for much of the data which led to the specification of Prospect Theory's value and decision weighing functions. The procedure also explains observed violations of stochastic dominance, transitivity, and invariance not accounted for in available alternatives to expected utility.

Key words: choice anomalies, expected utility, similarity judgments

\section{Introduction}

Given a choice between risky alternatives differing substantially in expected value, individual behavior appears fairly coherent. On the other hand, for alternatives which are at least proportionally close in expected value, violations of the axioms of the expected utility hypothesis occur. Two explanations for such rationality in the large, but irrationality in the small, come to mind. First, cognitive and experiential limitations may force agents to base their choices on crude approximations to their true underlying preferences. If so, then appeal to preferences for the purposes of making choices may only be informative for options sufficiently disparate in utility. Alternatively, there may be decision costs associated with accurately and precisely evaluating the expected utilities of alternatives. Here, should a cursury evaluation of alternatives fail to reveal one as superior, agents may choose to base their decisions upon other criteria rather than incur the costs associated with more precise appraisal of the options.

Either of these possibilities suggests that observed irrationalities in the small may reflect characteristics of the procedures which agents employ to make choices when appeal to preference is uninformative. This interpretation of choice anomalies as "process revealing" was recently explored by Rubinstein (1988) in the context of common ratio violations of the independence axiom. The choice of $\mathrm{B}_{\text {over }} \mathrm{A}$, but $\mathrm{A}^{\prime}$ over $\mathrm{B}^{\prime}$, in the 
lotteries shown below is an example.
$\mathrm{A}:\{\$ 3000, .02 ; \$ 0, .98\}$
$\mathrm{A}^{\prime}:\{\$ 3000, .90 ; \$ 0, .10\}$
B: $\{\$ 6000, .01 ; \$ 0, .99\}$
$\mathrm{B}^{\prime}:\{\$ 6000, .45 ; \$ 0, .55\}$

Rubinstein proposed that, given such lotteries, agents first compare their nonzero prizes ( $\$ 3000$ versus $\$ 6000)$ and corresponding probabilities (.02 versus .01 or .90 versus .45 ) to see whether one alternative dominates the other. If not, agents repeat the comparisons in terms of the similarity/dissimilarity of the prizes and their probabilities. For lotteries judged to be similar on one of these dimensions, but dissimilar on the other, the dissimilar dimension becomes decisive in determining the choice. In the choice between $\mathrm{A}$ and $\mathrm{B}$, for example, the latter will appear preferable to the extent that it offers a dissimilar and better prize ( $\$ 6000$ versus $\$ 3000)$ at similar probability (.01 versus .02). Choice between lotteries judged to be similar or dissimilar on both dimensions (as in $\mathrm{A}^{\prime} \mathrm{B}^{\prime}$, if the probabilities, .90 and .45 , as well as the prizes, appear dissimilar in value) is assumed to be resolved in an unspecified third step. Rubinstein (1988) inquires as to what preferences would be consistent with such a three-step decision procedure.

This article examines the implications of these types of comparative decision rules for lottery pairs where each lottery involves $n \geq 2$ prizes. However, in contrast to Rubinstein (1988), and consistent with the observation that behavior appears rational in the large, agents are assumed to resort to such rules only in instances where appeal to preference has proven uninformative. As such, Step 1 in the decision scheme proposed in section 2 of this article is "appeal to preference," where, for alternatives differing sufficiently in expected utility, the process terminates. Otherwise, agents proceed to Step 2 and compare prizes and probabilities across alternatives in terms of their equality/inequality. However, agents not only compare the first prizes and corresponding probabilities in each lottery as in Rubinstein (e.g., the nonzero prizes and their probabilities in $\mathrm{AB}$ or $\mathrm{A}^{\prime}$ $\mathrm{B}^{\prime}$ ), but also the second prizes and their probabilities (e.g., $\$ 0$ with $\$ 0$ and .99 with .98 in $\mathrm{AB}$ ), and so forth, for a total of $n$ pairs of comparisons. For each pair, agents note whether the prizes involved are both "good" outcomes, both "bad" outcomes, or one a "good" outcome and the other a "bad" outcome. They then conclude whether each comparison of prizes and their corresponding probabilities "favors" the choice of one lottery over another (e.g., if one lottery offers a larger good outcome at greater probability), is "inconclusive" (e.g., one lottery offers a better outcome but the other offers a good outcome at higher probability), or is "inconsequential" (i.e., the comparisons involve identical prizes at identical probabilities). If one lottery is favored in any of these paired comparisons and inconsequential in the remainder, it is selected. Otherwise, agents proceed to a third step in the decision procedure.

In Step 3, agents repeat the set of comparisons from Step 2 in terms of the similarity/ dissimilarity of prizes and probabilities. Again, each pair of comparisons is judged to favor one lottery over the other (e.g., if one lottery offers a dissimilar and greater good outcome at similar probability), to be inconclusive (e.g., if one lottery offers a dissimilar and greater good outcome but the other offers a good outcome at dissimilar and greater probability), or to be inconsequential (i.e., both lotteries offer similar prizes at similar probabilities). Once all $n$ conclusions are reached, agents choose one lottery over the 
other if it is favored in any paired comparison and the alternative is not favored in any paired comparison (i.e., the remainder of comparisons are either inconsequential or inconclusive), and at random otherwise.

In expected utility, choices will be invariant to simple redescriptions of the available alternatives. Because the similarity model presented in section 2 involves inter-lottery comparisons, this will not necessarily be the case. Instead, the predictions of the model depend upon which prizes and probabilities agents compare across alternatives-decisions which can be influenced by how the alternatives are represented. The choices of expected utility maximizers will also be invariant to certain arithmetic manipulations which alter the proximity of prizes or probabilities across lotteries. Because similarity judgments among prizes and probabilities may be intransitive (e.g., in comparisons of $\$ 10, \$ 7$, and $\$ 5, \$ 10$ may appear similar to $\$ 7, \$ 7$ similar to $\$ 5$, but $\$ 10$ dissimilar to $\$ 5$ ), choices recommended by such judgments will not be invariant to these manipulations. Predictions following from these features of the model are presented in sections 3 through 5 and compared with those of two of the more descriptively powerful alternatives to expected utility, the regret theory of Loomes and Sugden (1982) and Kahneman and Tversky's (1979) prospect theory.

Regret theory predicts rational violations of equivalence, ${ }^{1}$ monotonicity, ${ }^{2}$ and transitivity in choices between statistically dependent alternatives-predictions largely borne out experimentally. Section 3 shows that the similarity model also predicts such violations and predicts that they will occur among statistically independent as well as dependent alternatives. Consistent with the latter prediction, section 4 reviews evidence revealing violations of monotonicity between statistically independent alternatives. Section 4 also presents additional predictions regarding violations of transitivity which have no analogs in regret theory. Specifically, the intransitive pattern of responses reported by Tversky (1969) is shown to occur when agents perceive similarities between probabilities across alternatives, while the opposite pattern of intransitivities is predicted when there are similarities among the prizes. Experimental results confirming this last result are presented.

Section 5 of the article considers implications of the similarity model regarding evidence upon which Kahneman and Tversky's (1979) prospect theory was based. Properties of that theory's decision weight function and value function were posited based largely upon evidence revealing violations of the independence axiom (e.g., the choice of $B$ and $A^{\prime}$ discussed earlier) and upon evidence revealing that the pattern of choices reverses when the prizes involved are losses instead of gains. ${ }^{3}$ Prospect theory's isolation effect, on the other hand, followed from evidence indicating that individuals exhibiting risk-seeking behavior in choices between simple lotteries like $\mathrm{AB}$ exhibit risk-averse behavior when the alternatives are presented in a two-stage game form, in violation of the reduction of compound lotteries axiom. Section 5 shows that violations of the independence axiom, reflection effects, and violations of the reduction of compound lotteries axiom also follow as a consequence of similarity judgments. Reasoning identical to that implying violations of the reduction of compound lotteries axiom also implies that agents will exhibit systematic preference reversals among different two-stage representations of the same pair of probability distributions of outcomes. Experimental evidence supporting this prediction and suggesting that such behavior is better explained by similarity judgments than by prospect theory is reviewed. 


\section{A Model of generalized similarity judgments}

This section presents a model of choice in which agents employ closely related and increasingly liberal comparative evaluation procedures to make decisions when they are unable or unwilling to discriminate between alternatives in terms of their expected utilities. For purposes of operationalizing agents' inability or unwillingness to discriminate between alternatives close in value, assume that they evaluate risky alternatives according to an expected utility model incorporating a threshold or just noticeable difference parameter, $\delta .{ }^{4}$

Consider lotteries $\mathrm{L}_{1}$ and $\mathrm{L}_{2}$, where for $i=1,2$ and $j=1,2, \ldots, n, x_{i j} \in[0,1], p_{i j}>0$, and $\Sigma_{j} p_{i j}=1$.

$$
\begin{aligned}
& \mathrm{L}_{1}=\left\{x_{11}, p_{11} ; x_{12}, p_{12} ; \ldots, x_{1 n}, p_{1 n}\right\} \\
& \mathrm{L}_{2}=\left\{x_{21}, p_{21} ; x_{22}, p_{22} ; \ldots, x_{2 n}, p_{2 n}\right\}
\end{aligned}
$$

For the purpose of choosing between such alternatives, assume that agents employ the following three-step decision scheme:

Step 1: Appeal to preference (i.e., compute the expected utilities of the alternatives). If $\mathrm{EU}\left(\mathrm{L}_{1}\right)+\delta>\mathrm{EU}\left(\mathrm{L}_{2}\right)$ then $\mathrm{L}_{1}$ is preferred to $\mathrm{L}_{2}$. Otherwise, go to Step 2.

Step 2: Compare prizes $x_{1 j}$ and $x_{2 j}$ and probabilities $p_{1 j}$ and $p_{2 j}$ in terms of their equality or inequality for $j=1$ to $n$.

Note that for each comparison of prizes, whether it involves a comparison of good outcomes, bad outcomes, or a good and a bad outcome, good and bad are defined as follows:

A prize $x_{i j}$ is a good prize if a) it is the best possible outcome in either lottery, or

b) it is greater than zero or equal to zero and not the worst possible outcome in either lottery.

A prize $x_{i j}$ is a bad prize if a) it is the worst possible outcome in either lottery, or

b) it is less than zero and not the best possible outcome in either lottery.

Conclude for each of the $n$-paired comparisons whether it favors $\mathrm{L}_{1}\left(\mathrm{~L}_{2}\right)$, is inconclusive, or is inconsequential; these conclusions depend upon whether the prizes compared are both defined as good outcomes, bad outcomes, or one good and one bad. Considering first the case of unequal outcomes, if the prizes are both good, the greater one will be favored at greater than or equal probability. If one prize is greater than the other but the other is to occur with greater probability, the paired comparison is inconclusive-one lottery offers a more desirable prize, but the other offers a desirable prize at higher probability. If the two prizes are both bad outcomes, the greater (less undesirable) outcome will instead be favored at less than or equal probability. At greater probability, the result is inconclusive-the lottery offering the less undesirable prize, nonetheless, does so at higher probability. Finally, in comparisons of a good and bad prize, in which 
case the former must be greater than the latter, the paired comparison favors the lottery offering the good outcome at any positive probability.

If, instead, prizes compared are equal, then, in comparisons of good prizes, higher probability is desirable; whereas for comparisons of bad prizes, lower probability is desirable. If probabilities as well as prizes are equal, then the comparison is deemed inconsequential.

Once conclusions are reached regarding all $n$ pairs of comparisons:

Choose $\mathrm{L}_{1}\left(\mathrm{~L}_{2}\right)$ if it is favored in any comparisons and inconsequential in the remainder. Otherwise, go to Step 3.

For the purposes of specifying Step 3, assume the binary relations $>_{x}$ and $>_{p}$, reading "greater than and dissimilar," are strict partial orders (asymmetric and transitive) ${ }^{5}$ on consequences and probabilities, respectively. As such, the similarity relations $\sim^{x}$ and $\sim^{p}$, defined by $>_{x}$ and $>_{p}$, are symmetric ${ }^{6}$ but not necessarily transitive, in that for some prizes $x_{f}$ $>x_{g}>x_{h}, x_{f} \sim{ }^{x} x_{g}, x_{g} \sim{ }^{x} x_{h}$, but $x_{f}>{ }^{x} x_{h}$, with the same being possible for probabilities.

Step 3: Compare prizes $x_{1 j}$ with $x_{2 j}$ and probabilities $p_{1 j}$ with $p_{2 j}$ in terms of their similarity or dissimilarity for $j=1$ to $n$.

Note for each comparison of prizes, whether it involves a comparison of good outcomes, bad outcomes, or a good and a bad outcome, where good and bad are defined as before. Conclude for each of the $n$ paired comparisons whether it favors $L_{1}\left(L_{2}\right)$, is inconclusive, or is inconsequential.

When one prize is perceived as dissimilar and greater than the other, these conclusions are analogous to those in Step 2. Specifically, the lottery offering the greater and dissimilar prize will be favored at greater and dissimilar or similar probability when the prizes compared are good, favored at less than and dissimilar or similar probability when the prizes are bad, and favored at any probability when the prize offered is a good and is compared with a bad. Otherwise, the pair of comparisons will be deemed inconclusive.

For prizes perceived as similar, conclusions are again entirely analogous to those in Step 2 for comparisons of equal good or bad prizes. Here, however, a good and a bad prize can be perceived as similar. If so, and the good prize involved is also the best possible outcome in either lottery, then the lottery containing a greater and dissimilar probability will be favored (the best outcome or something similar to it is desirable at noticeably higher probability). Conversely, if the bad outcome is also the worst possible outcome, the lottery containing a lesser and dissimilar probability will be favored (for the worst outcome or something similar to it, noticeably lower probability is desirable). Otherwise, the pairs are either inconsequential (i.e., if both prizes and probabilities are similar) or inconclusive (i.e., if the good prize is not the best possible nor the bad prize the worst possible).

Once conclusions are reached regarding all $n$ pairs of comparisons:

Choose $\mathrm{L}_{1}\left(\mathrm{~L}_{2}\right)$ if it is favored in any paired comparisons and $\mathrm{L}_{2}\left(\mathrm{~L}_{1}\right)$ is not favored in any (i.e., the remaining comparisons are either inconsequential or inconclusive), and at random otherwise. 
With the model of choice specified, we now consider how it explains various types of choice anomalies and, in the process, provides a reinterpretation of two of the more powerful alternatives to expected utility-namely, Loomes and Sugden's (1982) regret theory, and Kahneman and Tversky's (1979) prospect theory.

\section{Implications consistent with regret theory}

\subsection{Juxtaposition effects}

Consider the following 3-tuple representations, denoted RIa and RIb, of common ratio type choices between probability distributions $\mathrm{R}\left(\right.$ isky): $\left\{x_{1}, \lambda p ; x_{3}, 1-\lambda p\right\}$ and $S$ (afe): $\left\{x_{2}\right.$, $\left.p ; x_{3}, 1-p\right\}$, for values of $x_{1}>x_{2}>x_{3} \geq 0\left(0 \geq x_{3}>x_{2} \geq x_{1}\right),{ }^{7} 1>\lambda>0,1>p>$ 0 , and $1-p-\lambda p>0$.

RIa

$\mathrm{Ra}:\left\{x_{1}, \lambda p ; x_{3}, p-\lambda p ; x_{3}, 1-p\right\}$

$\mathrm{Sa}:\left\{x_{2}, \lambda p ; x_{2}, p-\lambda p ; x_{3}, 1-p\right\}$
RIb

$\mathrm{Rb}:\left\{x_{1}, \lambda p ; x_{3} p ; x_{3}, 1-p-\lambda p\right.$

$\mathrm{Sb}:\left\{x_{3}, \lambda p ; x_{2}, p ; x_{3}, 1-p-\lambda p\right\}$

Given either lottery pair, if choice is not resolved by appeal to preference, as will be assumed henceforth, agents will proceed to Step 2 and compare prizes and their corresponding probabilities in terms of their equality/inequality, beginning with those in the first column of the representation (e.g., $x_{1}$ with $x_{2}$ and $\lambda p$ with $\lambda p$ in RIa), then those in the second column (e.g., $x_{2}$ with $x_{3}$ and $p-\lambda p$ with $p-\lambda p$ in RIa), and then those in the third. Here, and henceforth, except where explicitly noted, these Step 2 comparisons will fail to recommend a choice (e.g., in RIa, the first paired comparison favors Ra, but the other two paired comparisons favor Sa), in which case agents proceed to Step 3. Here the same set of comparisons is repeated except in terms of the similarity/dissimilarity of prizes and probabilities. In RIa and RIb, probabilities are identical across matched prize-probability pairs and, as a consequence, will be deemed similar by the symmetry property of $\sim^{P}$. As such:

Prediction 1: For probability distributions R and S represented as RIa or RIb, similarity implies that choices will be independent of $p$ (i.e., there will be no common ratio effects).

Next note that for either type of representation, symmetry of $\sim^{x}$ as well as $\sim^{p}$ implies that the comparison of the prize-probability pair $\left(x_{3}, 1-p\right)$ with itself in RIa and $\left(x_{3}, 1-p\right.$ $-\lambda p$ ) with itself in RIb will always be inconsequential, in which case they cannot influence the choice made nor will changing the value of $x_{3}$ here influence the choice. As such:

Prediction 2: For probability distributions R and S represented as RIa or RIb, similarity implies that choices will be independent of the value of $x_{3}$ in the common component, $\left(x_{3}, 1-p\right)$ in RIa or $\left(x_{3}, 1-p-\lambda p\right)$ in RIb (i.e., there will be no common consequence effects). ${ }^{8}$ 
Next consider choosing between $\mathrm{Ra}$ and $\mathrm{Sa}$ and between $\mathrm{Rb}$ and $\mathrm{Sb}$ where $x_{1}>x_{2}>$ $x_{3} \geq 0$. Individuals for whom $x_{1}>^{x} x_{2}$ but $x_{2} \sim^{x} x_{3}$ will exhibit the choice pattern RaRb, as the first pair of comparisons in either representation will favor the riskier option with the remaining pairs of comparisons being inconclusive. Individuals for whom $x_{1} \sim^{x} x_{2}$ $>^{x} x_{3}$ will choose $\mathrm{Sa}$ by similarity with the choice between $\mathrm{Rb}$ and $\mathrm{Sb}$ resolved at random, producing choice patterns $\mathrm{SaRb}$ or $\mathrm{SaSb}$. The remaining possible pattern $\mathrm{RaSb}$, however, can never systematically occur as a consequence of similarity, since for $\mathrm{Sb}$ to be recommended by similarity, $x_{1}$ would have to appear similar to $x_{3}$ (but, if so, then both choices are resolved at random). For prizes ordered $0 \geq x_{3}>x_{2}>x_{1}$, on the other hand, it is $\mathrm{Rb}$ which can never be selected by similarity. As such:

Prediction 3: For choices between probability distributions $\mathrm{R}$ and $\mathrm{S}$, represented as RIa and RIb with $x_{1}>x_{2}>x_{3} \geq 0\left(0 \geq x_{3}>x_{2}>x_{1}\right)$, similarity implies not RaSb (not $\mathrm{SaRb}){ }^{9}$

\subsection{Violations of transitivity}

Consider probability distributions A: $\left\{x_{1}, p_{1} ; x_{4}, 1-p_{1}\right\}, \mathrm{B}:\left\{x_{2}, p_{2} ; x_{4}, 1-p_{2}\right\}$, and C: $\left\{x_{3}\right.$, $1\}$ for $x_{1}>x_{2}>x_{3}>x_{4} \geq 0$ and $1=p_{3}>p_{2}>p_{1}>0$. Suppose that agents are given choices between $A$ and $B, B$ and $C$, and $A$ and $C$, where the alternatives are represented or perceived by agents as shown in RII below where $\mathrm{p}_{\mathrm{I}}=\mathrm{p}_{1}, \mathrm{p}_{\mathrm{II}}=\mathrm{p}_{\mathrm{I}}+\mathrm{p}_{2}$ and $\mathrm{p}_{\mathrm{III}}=$ $\mathrm{p}_{1}-\mathrm{p}_{2}$.

\section{RII}
$\mathrm{A}:\left\{x_{1}, p_{\mathrm{I}} ; x_{4}, p_{\mathrm{III}} ; x_{4} p_{\mathrm{III}}\right\}$
$\mathrm{B}:\left\{x_{2}, p_{1} ; x_{2}, p_{\mathrm{III}} ; x_{4}, p_{\mathrm{III}}\right\}$
$\mathrm{A}:\left\{x_{1}, p_{1} ; x_{4}, p_{I I} ; x_{4}, p_{I I I}\right\}$
B: $\left\{x_{2}, p_{1} ; x_{2}, p_{I I} ; x_{4}, p_{\text {III }}\right\}$
C: $\left\{x_{3}, p_{1} ; x_{3}, p_{11} ; x_{3}, p_{\mathrm{III}}\right\}$
C: $\left\{x_{3}, p_{1} ; x_{3}, p_{\mathrm{II}} ; x_{3}, p_{\mathrm{III}}\right\}$

Denoting strict preference as $>$, individual choices between $\mathrm{A}$ and $\mathrm{B}, \mathrm{B}$ and $\mathrm{C}$, and $\mathrm{A}$ and $\mathrm{C}$ may reflect any of eight response patterns, two of which $\mathrm{A}>\mathrm{B}, \mathrm{B}>\mathrm{C}$, but $\mathrm{C}>\mathrm{A}$ (henceforth $\mathrm{ABC}$ ) and $\mathrm{B}>\mathrm{A}, \mathrm{C}>\mathrm{B}$, but $\mathrm{A}>\mathrm{C}$ (henceforth $\mathrm{BCA}$ ) are intransitive. ${ }^{10,11}$ In RII, like RIa and RIb, probabilities will be compared with themselves and deemed "similar," in which case choices will be determined either by similarities in the prizes or at random. The only choice consistent with the intransitive pattern $\mathrm{ABC}$ which can be fixed by similarity given these representations is $\mathrm{C}>\mathrm{A}$ if $x_{1} \sim^{x} x_{3}$, rendering the comparison of $x_{1}$ with $x_{3}$ and $p_{\mathrm{I}}$ with $p_{\mathrm{I}}$ inconsequential, but $x_{3}>^{x} x_{4}$, in which case the remaining two pairs of comparisons favor $\mathrm{C}$. Should $x_{1}$ appear similar to $x_{3}$, however, the choice pattern $\mathrm{B}>\mathrm{A}, \mathrm{C}>\mathrm{B}, \mathrm{C}>\mathrm{A}$ is implied (i.e., $x_{1} \sim^{x} x_{3}, x_{3}>^{x} x_{4} \Rightarrow x_{1} \sim^{x} x_{2}, x_{2}$ $>^{x} x_{4}$ and $x_{2} \sim^{x} x_{3}, x_{3}>^{x} x_{4}$ ). The alternative intransitive pattern, on the other hand, may occur if any of the following similarity relationships hold where blanks in the patterns indicate choices resolved at random:

$$
\begin{aligned}
& x_{1} \sim^{x} x_{2}, x_{2}>^{x} x_{4} \\
& x_{2} \sim^{x} x_{3}, x_{3}>^{x} x_{4} \\
& x_{1} \sim^{x} x_{2}, x_{2} \sim^{x} x_{3}, x_{3}>^{x} x_{4}, \text { but } x_{1}>^{x} x_{3}
\end{aligned}
$$

then patterns $B$ then patterns _, $\mathrm{C}$, then patterns $\mathrm{B}, \mathrm{C}$, 
If the prizes are reflected around zero (i.e., $0 \geq x_{4}>x_{3}>x_{2}>x_{1}$ ), this reasoning exactly reverses. As such:

Prediction 4: For RII representations of the choices between $\mathrm{A}$ and $\mathrm{B}, \mathrm{B}$ and $\mathrm{C}$, and $\mathrm{A}$ and $\mathrm{C}$, where $x_{1}>x_{2}>x_{3}>x_{4} \geq 0\left(0 \geq x_{4}>x_{3}>x_{2}>x_{1}\right)$, similarity $\Rightarrow$ not $\mathrm{ABC}$ $(\mathrm{BCA}){ }^{12}$

\subsection{Violations of equivalence and monotonicity}

Consider the following 3-tuple representation, denoted RIII, of a choice between the probability distribution $\left[x_{1}, 1 / 3 ; x_{2}, 1 / 3 ; x_{3}, 1 / 3\right]$ and itself.

$$
\begin{aligned}
& \text { RIII } \\
& \text { A: }\left\{x_{1}, 1 / 3 ; x_{2}, 1 / 3 ; x_{3}, 1 / 3\right\} \\
& \text { A }^{\prime}:\left\{x_{3}, 1 / 3 ; x_{1}, 1 / 3 ; x_{2}, 1 / 3\right\}
\end{aligned}
$$

Given this choice, $\mathrm{A}^{\prime}$ can never be systematically selected based upon similarity judgments, because for this to occur it would have to be that the comparison of $x_{1}$ with $x_{3}$ and $1 / 3$ with $1 / 3$ was inconsequential, requiring $x_{1} \sim^{x} x_{3}$. Were this so, however, then all three pairs of comparisons would yield inconsequential results, in which case the choice would be resolved at random (i.e., $x_{1} \sim^{x} x_{3} \Rightarrow x_{1} \sim^{x} x_{2}$ and $x_{2} \sim^{x} x_{3}$ ). If, however, $x_{1}>{ }^{x} x_{3}$, then, if $x_{1} \sim^{x} x_{2}$ and $x_{2} \sim^{x} x_{3}$, A will appear preferable, since it is favored in the first comparison $\left(x_{1}\right.$ versus $\left.x_{3}\right)$, with the remaining comparisons being inconsequential. Moreover, we may be able to add a sufficiently small amount $\varepsilon$ to $x_{2}$ in $\mathrm{A}^{\prime}$ without altering the similarity perception (i.e., obtain $x_{2}+\varepsilon-^{x} x_{3}$ ), in which case agents may systematically violate monotonicity. As such:

Prediction 5: For choices between either equivalent lotteries or a stochastically dominating and dominated alternative represented as in RIII, similarity $\Rightarrow$ not $\mathrm{A}^{\prime} .{ }^{13}$

\subsection{Predictions according to regret theory}

Behaviors corresponding closely to those in Predictions 1,2,3,4, and 5 also follow in the regret theory of Loomes and Sugden (1982), assuming that the lotteries involved are statistically dependent. To see why, let $\mathrm{S}:\left\{\mathrm{S}_{1}, \mathrm{~S}_{2}, \ldots \mathrm{S}_{n}\right\}$ be the set of possible states of the world where each state $\mathrm{S}_{j}$ occurs with probability $p_{j}\left(\sum_{j} p_{j}=1\right)$. Let $\mathrm{A}_{1}$ and $\mathrm{A}_{2}$ be two actions where agents choosing $A_{1}\left(A_{2}\right)$ receive outcome $x_{1 j}\left(x_{2 j}\right)$ if state $S_{j}$ occurs $(j=1$ to $n$ ). Let $\sim$ and $\geq$ denote indifference and weak preference, respectively. In regret theory, agents choose between actions according to the decision rule $A_{1} \gtreqless A_{2} \Leftrightarrow$ $\sum_{j} p_{j} \psi\left(x_{i j}, x_{k j}\right)>=<0$ where $\psi(.,$.$) is a real valued regret/rejoice function assumed to$ exhibit the following three properties:

1. Skew-symmetry: For all $x_{g}, x_{h}: \psi\left(x_{g}, x_{h}\right)=-\psi\left(x_{h}, x_{g}\right)$; 
2. Increasingness: For all $x_{f}, x_{g}, x_{h} \in \mathrm{X}: \psi\left(x_{f}, x_{g}\right)>=<0 \Leftrightarrow \psi\left(x_{f}, x_{h}\right)>=<\psi\left(x_{g}, x_{h}\right)$; and 3. "Regret aversion" or "convexity": For all $x_{f}, x_{g}, x_{h} \in X: \psi\left(x_{f}, x_{g}\right)>0$ and $\psi\left(x_{g}, x_{h}\right)>$ 0 and $\psi\left(x_{f}, x_{h}\right)>0 \Leftrightarrow \psi\left(x_{f}, x_{h}\right)>\psi\left(x_{f}, x_{g}\right)+\psi\left(x_{g}, x_{h}\right)$.

According to regret theory, choices between $\mathrm{Ra}$ and $\mathrm{Sa}$ and between $\mathrm{Rb}$ and $\mathrm{Sb}$ where the alternatives are interpreted as actions will be resolved as follows:

$$
\begin{aligned}
& \mathrm{Ra}^{>} \sim<\mathrm{Sa} \Leftrightarrow(\lambda p) \psi\left(x_{1}, x_{2}\right)+(p-\lambda p) \psi\left(x_{3}, x_{2}\right)+(1-p) \psi\left(x_{3}, x_{3}\right)>=<0 \\
& \mathrm{Rb}^{>} \sim<\mathrm{Sb} \Leftrightarrow(\lambda p) \psi\left(x_{1}, x_{3}\right)+p \psi\left(x_{3}, x_{2}\right)+(1-p-\lambda p) \psi\left(x_{3}, x_{3}\right)>=<0 .
\end{aligned}
$$

The term $\psi\left(x_{3}, x_{3}\right)$ in each expression equals zero by skew-symmetry (thus, no common consequence effects). The probability $p$ can be factored out of the remaining two terms in each expression (thus, no common ratio effects). Given these simplifications, the choice of $\mathrm{Ra}$ over Sa requires that $\lambda p \geq \psi\left(x_{1}, x_{2}\right) /\left[\psi\left(x_{1}, x_{2}\right)+\psi\left(x_{3}, x_{2}\right)\right]$. The choice of $\mathrm{Sb}$ over $\mathrm{Rb}$ requires that $\psi\left(x_{1}, x_{2}\right) / \psi\left(x_{1}, x_{3}\right) \geq \lambda p$. Regret aversion implies $\psi\left(x_{1}, x_{2}\right) /$ $\left[\psi\left(x_{1}, x_{2}\right)+\psi\left(x_{3}, x_{2}\right)\right]>\psi\left(x_{1}, x_{2}\right) / \psi\left(x_{1}, x_{3}\right)$, ruling out the choice pattern RaSb.

Regarding systematic violations of transitivity, individuals choosing according to regret theory will evaluate choices between $\mathrm{A}$ and $\mathrm{B}, \mathrm{B}$ and $\mathrm{C}$, and $\mathrm{C}$ and $\mathrm{A}$, again interpreted as choices between actions, as follows:

$$
\begin{aligned}
& \mathrm{A}^{>} \sim<\mathrm{B} \Leftrightarrow \operatorname{pI} \psi\left(x_{1}, x_{2}\right)+\mathrm{p}_{\text {II }} \psi\left(x_{4}, x_{2}\right)+\operatorname{p} \text { III } \psi\left(x_{4}, x_{4}\right)>=<0, \\
& \mathrm{~B}>\sim<\mathrm{C} \Leftrightarrow \operatorname{pI} \psi\left(x_{2}, x_{3}\right)+\operatorname{p}_{I I} \psi\left(x_{2}, x_{3}\right)+\operatorname{p}_{\text {III }} \psi\left(x_{4}, x_{3}\right)>=<0, \\
& \mathrm{C}^{>} \sim<\mathrm{A} \Leftrightarrow \operatorname{pI} \psi\left(x_{3}, x_{1}\right)+\operatorname{p}_{\text {II }} \psi\left(x_{3}, x_{4}\right)+\operatorname{p}_{\text {III }} \psi\left(x_{3}, x_{4}\right)>=<0 .
\end{aligned}
$$

Exploiting the skew-symmetry property of the $\psi(.,$.$) function and rearranging terms,$ the $\mathrm{ABC}$ pattern occurs if the following expression is positive, whereas the $\mathrm{BCA}$ opattern occurs if it is negative:

$$
\begin{aligned}
& \mathrm{p}_{\mathrm{I}}\left[\psi\left(x_{1}, x_{2}\right)+\psi\left(x_{2}, x_{3}\right)-\psi\left(x_{1}, x_{3}\right)\right]+\mathrm{p}_{\mathrm{II}}\left[\psi\left(x_{2}, x_{3}\right)+\psi\left(x_{3}, x_{4}\right)-\psi\left(x_{2}, x_{4}\right)\right] \\
& +\mathrm{p}_{\mathrm{III}}\left[\psi\left(x_{3}, x_{4}\right)+\psi\left(x_{4}, x_{4}\right)-\psi\left(x_{3}, x_{4}\right)\right] .
\end{aligned}
$$

Skew symmetry implies that the third term equals zero, while the convexity property of $\psi(. .$.$) implies that the first and second terms are negative. As such, individuals choosing$ according to regret theory may exhibit the intransitive pattern $\mathrm{BCA}$, but never the pattern ABC.

Finally, agents behaving according to regret will choose between actions $A$ and $A^{\prime}$ in RIII (both corresponding to the same probability distribution) as $A>\sim<A^{\prime} \Leftrightarrow$ $1 / 3\left[\psi\left(x_{1}, x_{3}\right)-\psi\left(x_{1}, x_{2}\right)-\psi\left(x_{2}, x_{3}\right)\right]^{>}=<0$. Regret aversion implies that this expression is positive so individuals will exhibit a strict preference for $A .{ }^{14}$ To the extent that adding a sufficiently small amount to $x_{2}$ in $\mathrm{A}^{\prime}$ does not alter this inequality, also a possibility given the convexity of $\psi(.,$.$) , agents may also prefer A$ when it is stochastically dominated by $A^{\prime}$.

These correspondences between the predictions of regret theory and those following from the hypothesis that choices are based, in part, upon similarity judgments are not 
accidental. The absence of common ratio effects implied by regret follows from similarity judgments if the lotteries are represented such that probabilities across lotteries are compared with themselves as a consequence of the symmetry property of $\sim^{p}$, while the absence of common consequence violations follows from the symmetry of $\sim^{x}$ as well as $\sim^{p}$. Likewise, behaviors implied by the assumptions that $\psi(.,$.$) is increasing and convex$ (i.e., for $\left.x_{1}>x_{2}>x_{3}, \psi\left(x_{1}, x_{3}\right)>\psi\left(x_{1}, x_{2}\right)+\psi\left(x_{2}, x_{3}\right)\right)$ follow, in some cases in weaker form, ${ }^{15}$ from the assumption that the similarity relation, $\sim^{x}$, is not necessarily transitive (i.e., for some $x_{1}>x_{2}>x_{3}, x_{1} \sim^{x} x_{2}, x_{2} \sim^{x} x_{3}$, but $x_{1}>^{x} x_{3}$ ). These technical coincidences do not, however, imply that the predictions of the models are indistinguishable, as the following evidence and predictions reveal.

\section{Implications of inconsistent with regret theory}

\subsection{Violations of monotonicity, again}

Tversky and Kahneman (1986) presented subjects with a choice between Options A and $B$ below, where the probabilities of winning each prize in each lottery were described by the percentage of marbles of different colors in different boxes.

$\begin{array}{llllll}\text { Option A } & \begin{array}{l}90 \% \text { white } \\ \$ 0\end{array} & \begin{array}{l}6 \% \text { red } \\ \text { win } \$ 45\end{array} & \begin{array}{l}1 \% \text { green } \\ \text { win } \$ 45\end{array} & \begin{array}{l}1 \% \text { blue } \\ \text { lose } \$ 10\end{array} & \begin{array}{l}2 \% \text { yellow } \\ \text { lose } \$ 15\end{array} \\ \text { Option B } & \begin{array}{l}90 \% \text { white } \\ \$ 0\end{array} & \begin{array}{l}6 \% \text { red } \\ \text { win } \$ 45\end{array} & \begin{array}{l}1 \% \text { green } \\ \text { win } \$ 30\end{array} & \begin{array}{l}1 \% \text { blue } \\ \text { lose } \$ 15\end{array} & \begin{array}{l}2 \% \text { yellow } \\ \text { lose } \$ 15\end{array}\end{array}$

Simple comparison of the lotteries by column reveals that $\mathrm{A}$ stochastically dominates B, a fact recognized by all 88 of Tversky and Kahneman's (1986) subjects. They offered an additional 124 subjects a choice between $\mathrm{A}^{\prime}$ and $\mathrm{B}^{\prime}$, shown below with the probabilities of winning each prize in each lottery, again described by the percentage of marbles of different colors in different boxes.

$\begin{array}{lllll}\text { Option } \mathrm{A}^{\prime} & \begin{array}{l}90 \% \text { white } \\ \$ 0\end{array} & \begin{array}{l}7 \% \text { red } \\ \text { win } \$ 45\end{array} & \begin{array}{l}1 \% \text { green } \\ \text { lose } \$ 10\end{array} & \begin{array}{l}2 \% \text { yellow } \\ \text { lose } \$ 15\end{array} \\ \text { Option } \mathrm{B}^{\prime} & \begin{array}{l}90 \% \text { white } \\ \$ 0\end{array} & \begin{array}{l}6 \% \text { red } \\ \text { win } \$ 45\end{array} & \begin{array}{l}1 \% \text { green } \\ \text { win } \$ 30\end{array} & \begin{array}{l}3 \% \text { yellow } \\ \text { lose } \$ 15\end{array}\end{array}$

Given this choice, a majority of subjects $(72 / 124$ or $58 \%)$ preferred lottery $\mathrm{B}^{\prime}$. A comparison of lottery $\mathrm{A}^{\prime}$ with $\mathrm{A}$, and $\mathrm{B}^{\prime}$ with $\mathrm{B}$, however, reveals that the latter are simply reformulations of the former. ${ }^{16}$ As such, $58 \%$ of subjects violate stochastic dominance when lotteries $\mathrm{A}$ and $\mathrm{B}$ are reformulated as $\mathrm{A}^{\prime}$ and $\mathrm{B}^{\prime}$.

To the extent that choices between $\mathrm{A}$ and $\mathrm{B}$, and between $\mathrm{A}^{\prime}$ and $\mathrm{B}^{\prime}$ are statistically independent, regret theory incorrectly predicts adherence to dominance in both cases. 
Both choices are, on the other hand, entirely consistent with the decision procedure proposed in this article. To resolve the choice between $A$ and $B$ or $A^{\prime}$ and $\mathrm{B}^{\prime}$, agents would first appeal to preference. Given the small (20 cent) difference in expected values between alternatives, this procedure might quite reasonably be expected to be uninformative. If so, agents would proceed to Step 2, comparing prizes and probabilities across alternatives in terms of their equality or inequality. In the choice between A and B, A would be favored in the third paired comparison $(\$ 45>\$ 30$ and $1 \%=1 \%)$ as well as the fourth $(-\$ 10>-\$ 15$ and $1 \%=1 \%)$, with the remaining paired comparisons (all of which involve identical prizes at identical probabilities) being inconsequential. As a consequence, the dominant alternative $A$ would be selected. In the choice between $\mathrm{A}^{\prime}$ and $\mathrm{B}^{\prime}$, on the other hand, comparisons in Step 2 will prove uninformative since the second $(\$ 45=\$ 45$ and $7 \%>6 \%)$ favors $A^{\prime}$, while the third $(\$ 30>-\$ 10$ and $1 \%=$ $1 \%)$ and fourth $(-\$ 15=-\$ 15$ and $3 \%>2 \%)$ favor $\mathrm{B}^{\prime}$. In this case, agents would proceed to Step 3 and repeat the set of comparisons in terms of the similarity/dissimilarity of prizes and their associated probabilities. Here, if the $7 \%$ and $6 \%$ chances of winning $\$ 45$ and the $2 \%$ and $3 \%$ chances of losing $\$ 15$ are deemed "similar," $\mathrm{B}^{\prime}$ will appear preferable so long as winning $\$ 30$ is perceived as dissimilar to losing $\$ 10$.

\subsection{Violations of transitivity, again}

The findings reported above suggest, contrary to regret theory, that the statistical independence or dependence of lotteries may not be of import in explaining violations of tenets of expected utility like stochastic dominance. ${ }^{17}$ These results do not, however, in and of themselves, imply that such behaviors occur as a consequence of the way the representation of lotteries influences what is compared with what in terms of similarity and dissimilarity. Consider, however, choices between $\mathrm{A}$ and $\mathrm{B}, \mathrm{B}$ and $\mathrm{C}$, and $\mathrm{A}$ and $\mathrm{C}$, shown below with $x_{1}>x_{2}>x_{3}>x_{4} \geq 0$ and $1 \geq p_{3}>p_{2}>p_{1}>0$. Denote this representation RIV.
RIV
A: $\left[x_{1}, p_{1} ; x_{4}, 1-p_{1}\right]$
$\mathrm{B}:\left[x_{2}, p_{2} ; x_{4}, 1-p_{2}\right]$
$\mathrm{A}:\left[x_{1}, p_{1} ; x_{4}, 1-p_{1}\right]$
$\mathrm{B}:\left[x_{2}, p_{2} ; x_{4} 1-p_{2}\right]$
C: $\left[x_{3}, p_{3} ; x_{4}, 1-p_{3}\right]$
C: $\left[x_{3}, p_{3} ; x_{4}, 1-p_{3}\right]$

Note that for this representation of the choices, in contrast to RII in the previous section, probabilities across lottery components are no longer matched with themselves. Thus, choices may depend not only upon similarities among prizes but among probabilities as well. Suppose, in particular, that $x_{1}>^{x} x_{2}>^{x} x_{3}>^{x} x_{4}$. In this case, to produce the intransitive choice pattern BCA by similarity in the probabilities, it would have to be the case that in the comparison between $x_{1}$ and $x_{3}$ and between $p_{1}$ and $p_{3}$, A was preferred to C because $p_{3} \sim^{p} p_{1}$. In that case, A would be "favored" in this pair of comparisons with the second pair of comparisons, $x_{4}$ with $x_{4}$ and $1-p_{3}$ with $1-p_{1}$, being inconsequential. This, however, implies $p_{3} \sim^{p} p_{2}$ and $p_{2} \sim^{p} p_{1}$, producing the transitive choice pattern $\mathrm{A}$ $>\mathrm{B}, \mathrm{B}>\mathrm{C}, \mathrm{A}>\mathrm{C}$. Any of the following similarity relations among probabilities will, on 
the other hand, allow for the alternative intransitive pattern $\mathrm{ABC}$ to occur:

$$
\begin{array}{ll}
\text { if } p_{2} \sim^{p} p_{1}, p_{3}>^{p} p_{2} & \text { then patterns } \mathrm{A}, \overrightarrow{\mathrm{C}}, \text { - are possible. } \\
\text { if } p_{2}>^{p} p_{1}, p_{3} \sim \sim^{p} p_{2} & \text { then patterns } \_, \mathrm{B}, \ldots \text { are possible. }
\end{array}
$$

As such:

Prediction 6: For choices between lotteries $\mathrm{A}$ and $\mathrm{B}, \mathrm{B}$ and $\mathrm{C}$, and $\mathrm{A}$ and $\mathrm{C}$ as represented in RIV, if $x_{1}>^{x} x_{2}>^{x} x_{3}>^{x} x_{4}$, similarity $\Rightarrow$ not BCA.

If, instead, it is the probabilities that are perceived as dissimilar (i.e., $p_{3}>^{p} p_{2}>^{p} p_{1}$ ), then similarities in the prizes can never produce the intransitive pattern $\mathrm{ABC}$, as the only one of these choices which can be fixed by similarity is $\mathrm{C}>\mathrm{A}$ if $x_{1} \sim^{x} x_{3}$. Should this be the case, however, then $x_{1} \sim^{x} x_{2}$ and $x_{2} \sim^{x} x_{3}$ also follow, requiring the transitive choice pattern $\mathrm{B}>\mathrm{A}, \mathrm{B}>\mathrm{C}, \mathrm{C}>\mathrm{A}$. Any of the following similarity relationships will, however, allow for the intransitive BCA pattern.

$$
\begin{array}{ll}
\text { if } x_{2} \sim^{x} x_{1}, x_{3}>^{x} x_{2} & \text { then patterns } \mathrm{B}, \overrightarrow{\mathrm{C}},- \\
\text { if } x_{2}>^{x} x_{1}, x_{3} \sim^{x} x_{2} & \text { then patterns } \overrightarrow{\mathrm{C}},- \\
\text { if } x_{2} \sim^{x} x_{1}, x_{3} \sim^{x} x_{2}, \text { but } x_{1}>^{x} x_{3} & \text { then patterns } \mathrm{B}, \mathrm{C},-
\end{array}
$$

As such:

Prediction 7: For choices between lotteries $\mathrm{A}$ and $\mathrm{B}, \mathrm{B}$ and $\mathrm{C}$, and $\mathrm{A}$ and $\mathrm{C}$ as represented in RIV, if $p_{3}>^{p} p_{2}>^{p} p_{1}$, similarity $\Rightarrow$ not $\mathrm{ABC}$.

For prizes ordered $0 \geq x_{4}>x_{3}>x_{2}>x_{1}$, Predictions 6 and 7 are exactly reversed.

Tversky (1969) presented subjects with choices between lotteries A and B, B and C, C and $\mathrm{D}, \mathrm{D}$ and $\mathrm{E}$, and $\mathrm{A}$ and $\mathrm{E}$, shown below:
A: $[\$ 5.00,7 / 24 ; \$ 0,17 / 24]$
$(\mathrm{EV}=\$ 1.46)$
B: $[\$ 4.75,8 / 24 ; \$ 0,16 / 24]$
$(\mathrm{EV}=\$ 1.58)$
C: $[\$ 4.50,9 / 24 ; \$ 0,15 / 24]$
$(\mathrm{EV}=\$ 1.69)$
D: $[\$ 4.25,10 / 24 ; \$ 0,14 / 24]$
$(\mathrm{EV}=\$ 1.77)$
E: $[\$ 4.00,11 / 24 ; \$ 0,13 / 24]$
$(E V=\$ 1.83)$

He reported observing systematic intransitivities of the form $A>B, B>C, C>D, D$ $>\mathrm{E}$, but $\mathrm{E}>\mathrm{A}$ analogous to the $\mathrm{ABC}$ pattern in Prediction 6. In the experiment, choices were displayed on cards with the prizes presented in dollars and their associated probabilities represented by blacked-out, pie-shaped areas on otherwise white disks. These features of the design can be interpreted as encouraging the perception of prizes as dissimilar, but probabilities adjacent in value as similar, as required in Prediction 6. To 
the extent that this interpretation is correct, reversing Tversky's experimental procedure should produce the intransitive pattern consistent with Prediction 7. To examine this possibility, Leland (1992) presented 64 Carnegie Mellon undergraduates with choices between the lotteries A and B, B and C, and A and C, shown below.
A: $[\$ 12.08, .63 ; \$ 0, .37]$
B: $[\$ 10.88, .71 ; \$ 0, .29]$
$\mathrm{C}:[\$ 9.67, .79 ; \$ 0, .21]$
$\mathrm{EV}=\$ 7.61$
$\mathrm{EV}=\$ 7.72$
$\mathrm{EV}=\$ 7.64$

In the experiment, and in contrast to Tversky's design, however, it was the probabilities that were stated numerically with the prizes $(\$ 12.08, \$ 10.88$, and $\$ 9.67)$ represented as blackened-out fractions ( $10 / 24$ ths, $9 / 24$ ths, and $8 / 24$ ths) of otherwise gray circles, where in each case an entirely blackened-out circle was worth $\$ 29.00$. Each subject received a sheet of instructions about the choice task and was presented with choices between lotteries A and B, B and C, and A and C. ${ }^{18}$ All subjects were informed that one of them would be randomly selected to play the lottery he or she had chosen on one of the problems contained in the questionnaire. The choice patterns obtained are shown below. Consistent with Prediction 7, the intransitive BCA pattern occurs significantly more frequently than the $\mathrm{ABC}$ pattern (25\% versus $0 \%$ ) and, indeed, occurs almost as often as all other patterns ( $25 \%$ versus $27 \%$ ), save the transitive pattern $\mathrm{B}>\mathrm{A}, \mathrm{B}>\mathrm{C}, \mathrm{C}>\mathrm{A}$ at $48 \%$.

$\begin{array}{lllllllll}\text { Pattern } & \text { BBC } & \text { BBA } & \text { ABA } & \text { BCC } & \text { ACA } & \text { ACC } & \text { ABC } & \text { BCA } \\ \#(\%) & 3(5 \%) & 1(2 \%) & 6(9 \%) & 31(48 \%) 4(6 \%) & 3(5 \%) & 0(0 \%) & 16(25 \%)\end{array}$

\section{A reinterpretation of the evidence motivating prospect theory}

\subsection{The properties of prospect theory}

The types of behaviors discussed to this point - namely, violations of transitivity, monotonicity, and equivalence - have not, by and large, been those which have traditionally preoccupied researchers. Instead, systematic departures from the assumption of risk aversion, discussed early on by Friedman and Savage (1948), and violations of the independence axiom, originally discussed by Allais (1953), have been the focus of attention and, in particular, led Kahneman and Tversky (1979) to specify Prospect Theory in the way they did. Prospect Theory consists of three primary components: a decisionweighting function $\pi($.$) ; a value function V($.), exhibiting risk seeking for losses and risk aversion for gains; and a set of editing rules, a prominent one being the "isolation effect" (the tendency to "disregard components that alternatives share and focus upon the components that distinguish them").

The decision-weighting function is assumed to exhibit two key properties-subcertainty $(\pi(p)+\pi(1-p)<1)$, and subproportionality (for probabilities $p$ and $r p, 1>r>$ 0 , the ratio of their decision weights $\pi(r p) / \pi(p)$ is closer to 1 for low values of $p$ than 
otherwise). The assumption that $\pi$ exhibits subcertainty was made based upon the observed tendency of individuals to commit common consequence violations of independence, choosing, for example, S: $\{\$ 2400,1\}$ over R: $\{\$ 2400, .66 ; \$ 2500, .33 ; \$ 0, .01\}$ but R': $\{\$ 2500, .33 ; \$ 0, .67\}$ over $\mathrm{S}^{\prime}:\{\$ 2400, .34 ; \$ 0, .66\}$ (the latter pair of lotteries being obtained from the former by replacing the common component $(\$ 2400, .66)$ in the first pair with the common component $(\$ 0, .66))$. Kahneman and Tversky (1979) interpret the first choice as indicating that "people overweight outcomes that are considered certain, relative to those which are merely probable - a phenomenon labeled the certainty effect." 19

The assumption that the decision-weighting function, $\pi($.$) , exhibits subproportionality$ and assumptions regarding the nature of the value function, $\mathrm{V}($.$) , were, on the other$ hand, based upon the pattern of choices observed in common ratio problems of the type discussed early in this article and how that pattern reverses when the lotteries are reflected around zero. Specifically, the choice of $S^{\prime}:\{\$ 3000, .90 ; \$ 0, .10\}$ over $R^{\prime}:\{\$ 6000$, . $45 ; \$ 0, .55\}$, but $R^{\prime}:\{-\$ 6000, .45 ; \$ 0, .55\}$ over $S^{\prime}:\{-\$ 3000, .90 ; \$ 0, .10\}$ was interpreted as indicating that $\mathrm{V}($.$) was defined on gains and losses and S-shaped (risk-averse for$ gains and risk-seeking for losses). The tendency of individuals to exhibit the opposite risk-preference patterns when the probabilities associated with the nonzero outcomes were small (i.e., R: $\{\$ 6000, .01 ; \$ 0, .99\}>$ S: $\{\$ 3000, .02 ; \$ 0, .98\}$ and S: $\{-\$ 3000, .02 ; \$ 0, .98\}$ $>\mathrm{R}:\{-\$ 6000, .01 ; \$ 0, .99\})$ was taken to imply the subproportionality property of $\pi($.$) .$

Finally, the assumption that individuals tend to ignore components common across alternatives followed from behavior observed when subjects were given a two-stage game where in the first stage there was a .25 chance of going to the second stage and playing either the lottery $S^{\prime}:\{\$ 3000\}$ or the lottery $R^{\prime}:\{\$ 4000, .8 ; \$ 0, .2\}$. Here, agents required to choose between $\mathrm{R}^{\prime}$ and $\mathrm{S}^{\prime}$ before the game began tended to choose the safer alternative. When the alternatives were presented in single stage form (i.e., choose between $\{\$ 3000$, $.25 ; \$ 0, .75\}$ and $\{\$ 4000, .20 ; \$ 0, .80\}$ ), however, they chose the riskier one, in violation of the reduction of compound lotteries axiom.

Now consider how such behaviors might come about as a consequence of choice based upon similarity judgments.

\subsection{Common consequence violations of the independence axiom and reflection effects}

Consider again the choices $S R$ and $S^{\prime} R^{\prime}$, in which common consequence violations of the independence axiom occur:

$$
\begin{array}{ll}
\text { S: }\{\$ 2400,1 ; \$ 0, .0\} & S^{\prime}:\{\$ 2400, .34 ; \$ 0, .66\} \\
\text { R: }\{\$ 2400, .66 ; \$ 2500, .33 ; \$ 0, .01\} & \mathrm{R}:\{\$ 2500, .33 ; \$ 0, .67\}
\end{array}
$$

Note that, in the choice between the lottery $\mathrm{S}$ and $\mathrm{R}$, it is not obvious what agents will compare with what in terms of similarity. Any perceived representation of these choices involving a comparison of $\$ 2400$ and $\$ 0$ cannot, however, lead the agent to choose $R$ over $S$ by similarity, so long as $\$ 2400$ is greater than and dissimilar to $\$ 0$. If so, $\mathrm{R}$ offers some probability of the worst possible outcome. The choice between $\mathrm{S}^{\prime}:\{2400, .34 ; \$ 0, .66\}$ and 
$\mathrm{R}^{\prime}:\{\$ 2500, .33 ; \$ 0, .67\}$, on the other hand, will be resolved by similarity in favor of $\mathrm{R}^{\prime}$, so long as $\$ 2500>x \$ 2400$ and $.34 \sim^{p} .33$. As such, what is interpreted as a certainty effect in Prospect Theory occurs here, because, in the choice between $S$ and $R$, the fact that the latter offers some probability of the worst possible prize cannot be obscured. In the choice between $\mathrm{S}^{\prime}$ and $\mathrm{R}^{\prime}$, on the other hand, this is obscured. Indeed, along the lines of Prediction 3 (i.e., no common consequence violations) in section 3.1, similarity judgments imply that for representations of $S^{\prime}$ and $R^{\prime}$, shown below, the riskier alternative could not be selected based upon similarity, as the fact that it offers some probability of the worst possible outcome is made explicit.

$$
\begin{aligned}
& S^{\prime}:\{\$ 2400, .33 ; \$ 0, .66 ; \$ 2400, .01\} \\
& R^{\prime}:\{\$ 2500, .33 ; \$ 0, .66 ; \$ 0, .01\}
\end{aligned}
$$

Note also that should choices between $S$ and $R$ and between $S^{\prime}$ and $R^{\prime}$ involve losses instead of gains, similarity judgments imply reflection effects (i.e., $R:\{-\$ 2500, .33 ;-\$ 2400$, .66; $\$ 0$, $.01\}$ will be selected over $S:\{-\$ 2400,1\}$, and $S^{\prime}:\{-\$ 2400, .34 ; \$ 0, .67\}$ will be selected over $\mathrm{R}^{\prime}:\{-\$ 2500, .33 ; \$ 0, .67\}$.

\subsection{Common ratio effects, reflection, and violations of the reduction of compound lotteries axiom}

For purposes of explaining the remaining evidence discussed above, assumptions regarding the nature of the similarity relation, $\sim P$, are required. To see why, recall that Rubinstein (1988) showed that unless the relation, $\sim^{p}$, was of a ratio form such that $p_{1 j} \sim^{p} p_{2 j}$ if for $\delta>1,1 / \delta \leq p_{1 j} / p_{2 j} \leq \delta$, independence would be violated. Assume instead an $\varepsilon$-difference similarity such that $p_{1 j} \sim^{p} p_{2 j}$ if $\left|p_{1 j}-p_{2 j}\right| \leq \varepsilon$. Now consider common ratio type choices between probability distributions $\mathrm{R}$ and $\mathrm{S}$, represented as shown in $\mathrm{RV}$ for $x_{1}>x_{2}>x_{3} \geq 0$ :

$$
\begin{aligned}
& \text { RV } \\
& \text { R: }\left\{x_{1}, \lambda p ; x_{3}, 1-\lambda p\right\} \\
& \text { S: }\left\{x_{2}, p ; x_{3}, 1-p\right\}
\end{aligned}
$$

Given this representation, choice will be resolved as follows:

1. R by similarity if $x_{1}>^{x} x_{2}$ and $p \sim^{p} \lambda p ; x_{3} \sim^{x} x_{3}$ and $1-\lambda p \sim^{p} 1-p$, 2.S by similarity if $x_{1} \sim^{x} x_{2}$ and $p>p \lambda p ; x_{3} \sim^{x} x_{3}$ and $1-\lambda p>p 1-p$, 3. Either at random if $x_{1} \sim^{x} x_{2}$ and $p \sim p \lambda p ; x_{3} \sim^{x} x_{3}$ and $1-\lambda p \sim p 1-p$, or 4. S by similarity if $x_{1}>^{x} x_{2}$ and $p>^{p} \lambda p ; x_{3} \sim^{x} x_{3}$ and $1-\lambda p>^{p} 1-p$.

For reductions in the value of $p$, individuals initially holding perceptions corresponding to 1 will continue to choose $\mathrm{R}$, those corresponding to 2 eventually choose at random, and those corresponding to 3 will continue to choose at random. For agents choosing $\mathrm{S}$ 
because $\mathrm{R}$ offers the worst prize, $x_{3}$, at greater and dissimilar probability (case 4), on the other hand, reductions in $p$ sufficiently large to result in $p \sim p \lambda p$ (and $1-\lambda p \sim p 1-p$ ) will produce switches from choice of $\mathrm{S}$ to choice of $\mathrm{R}$. In no case, however, can reductions in $p$ ever result in switches from $\mathrm{R}$ to $\mathrm{S}$. Conversely, for the case of $0 \geq x_{3}>x_{2}>$ $x_{1}$, reductions in the value of $p$ cannot result in switches from $\mathrm{S}$ to $\mathrm{R}$. As such:

Prediction 8: For choices between probability distributions $\mathrm{R}$ and $\mathrm{S}$ for $x_{1}>x_{2}>x_{3}$ $\geq 0\left(0 \geq x_{3}>x_{2}>x_{1}\right)$, represented as RV, if $\sim^{p}$ is a difference similarity, then for decreases in $p$, and thus $p-\lambda p$, similarity $\Rightarrow$ not RS (not SR).

Now suppose instead that choices between $\mathrm{R}$ and $\mathrm{S}$ were represented in the two-stage game context in which reduction-of-compound-lotteries violations are revealed. Specifically, assume that choices between R: $\left\{x_{1}, r \lambda p ; x_{3}, 1-r \lambda p\right\}$ and $\mathrm{S}:\left\{x_{2}, r p ; x_{3}, 1-r p\right\}$ for $1 \geq p>0,1 \geq r \geq p$, and $1>\lambda>0$, presented as two-stage games, are represented or interpreted by agents as in RVI below:

$$
\begin{aligned}
& \text { RVI } \\
& \text { R: }\left[\left\{x_{1}, \lambda p ; x_{3}, 1-\lambda p\right\}, r ; x_{3}, 1-r\right], \\
& \text { S: }\left[\left\{x_{2}, p ; x_{3}, 1-p\right\}, r ; x_{3}, 1-r\right] .
\end{aligned}
$$

Note first that the comparison of the component $\left(x_{3}, 1-r\right)$ with itself will always be viewed as inconsequential. Note also that $r$ will always be perceived as similar to itself, in which case possible similarity relationships among the remaining prizes and their corresponding probabilities, as well as their implications regarding choice, are identical to those pertaining to Prediction 8. The value of $r$ does, however, indirectly influence the choice, in that the size of $r$ determines the difference $p-\lambda p$. For $r=p$, the difference $p$ $-\lambda p$ is maximal. As the value of $r$ is increased, the difference $p-\lambda p$ declines, in which case increases in $r$ have the same effect as reductions in $p$ in Prediction 8. As such:

Prediction 9: Given RVI representations of the choice between probability distributions $\mathrm{R}$ and $\mathrm{S}$, in which $r$ varies from low to high, if $\sim^{p}$ is a difference similarity, then for increases in $r$, similarity $\Rightarrow$ not RS (not SR for $0 \geq x_{3}>x_{2}>x_{1}$ ).

\subsection{Violations of invariance among two-stage lotteries}

As noted above, common ratio violations exhibited for gains and losses are interpreted in Prospect Theory as revealing information about risk preference and as indicating systematic biases in the way small probabilities are treated, while violations of the reduction-of-compound-lotteries axiom are interpreted as indicating an "isolation effect." Loomes and Sugden (1982) propose instead that common ratio violations occur, because, in the absence of contrary information, agents assume that the lotteries involved are statistically independent, in which case convexity of the regret/rejoice function $\psi(.,$.$) allows for such behavior. In the two-stage game format, on the other hand, they$ propose that, in the absence of specific information regarding the statistically dependence 
or independence of the alternatives, agents assume the former, in which case the less risky alternative may be preferred. Predictions 8 and 9 instead suggest that such behaviors are all just additional consequences of choice being based upon similarity judgments.

To discriminate between these alternative interpretations, note that, according to Prediction 9, violations of the reduction-of-compound-lotteries axiom are simply an extreme case of violations of invariance occurring among different two-stage representations of single-stage lotteries. Leland (1991) investigated this implication. Fifty Carnegie Mellon University undergraduates were presented with hypothetical choices between lotteries $\mathrm{R}_{i}$ and $\mathrm{S}_{i}$ for parameter values shown below.

Imagine being allowed to participate in the following two-stage game (for $i=1$ to 4 ). In the first stage, there is a $1-r_{i}$ chance that the game will end and you will win nothing, and an $r_{i}$ chance of moving to the second stage. If you reach the second stage, you will have a choice between either:

$\mathrm{R}_{i}$ : a lottery ticket offering a $\lambda p_{i}$ chance of winning $\$ 4000$, and a $1-\lambda p_{i}$ chance of winning nothing.

$S_{i}:$ a lottery ticket offering a $p_{i}$ chance of winning $\$ 3000$, and a $1-p_{i}$ chance of winning nothing.

If you must make a choice between $\mathrm{R}_{i}$ and $\mathrm{S}_{i}$ prior to the beginning of the game, which do you prefer?

\begin{tabular}{lcccccc}
\hline question & $r_{i}$ & $p_{i}$ & $\lambda p_{i}$ & $r_{i} p_{i}$ & $r_{i} \lambda p_{i}$ & $p_{i}-\lambda p_{i}$ \\
\hline 1 & .26 & .96 & .77 & .2496 & .200 & .19 \\
2 & .33 & .75 & .60 & .2475 & .198 & .15 \\
3 & .50 & .50 & .40 & .2475 & .198 & .10 \\
4 & .99 & .25 & .20 & .2475 & .198 & .05 \\
\hline
\end{tabular}

As is clear from the table, these four two-stage representations differ in the amount of mass placed in the first stage of the game (i.e., in the size of $r$ ) and in the difference in mass between the probabilities, $p$ and $\lambda p$, in the terminal lotteries $\mathrm{R}_{i}$ and $\mathrm{S}_{i}$. All four choice problems are, however, identical in the prizes and equivalent to the second decimal place in the probabilities. Assuming $\sim P$ is a difference similarity, the systematic occurrence of the choice patterns SSSS, SSSR, SSRR, SRRR, and RRRR is consistent with the model proposed in this article, while the systematic occurrence of the other 11 possible patterns is not.

The results for all 50 subjects are summaried in table 1 , where the 11 non-predicted response patterns and their associated frequencies of occurrence are presented first, followed by the five predicted patterns and their corresponding frequencies. Consistent with the prediction of the model, the latter account for $47 / 50(94 \%)$ of the response patterns observed, while the proportion of respondents choosing option $\mathrm{S}(90 \%, 86 \%$, 
Table 1 . Invariance violations

\begin{tabular}{llllll}
\hline & Q1 & Q2 & Q3 & Q4 & \# \\
$p_{j}-\lambda p_{i}=$ & .19 & .15 & .10 & .05 & responses \\
\hline 1 & non-predicted & patterns & $(=3 / 50)(6 \%))$ & & \\
2 & $\mathrm{R}$ & $\mathrm{R}$ & $\mathrm{S}$ & $\mathrm{S}$ & 0 \\
3 & $\mathrm{R}$ & $\mathrm{S}$ & $\mathrm{S}$ & $\mathrm{R}$ & 0 \\
4 & $\mathrm{R}$ & $\mathrm{S}$ & $\mathrm{S}$ & $\mathrm{S}$ & 0 \\
5 & $\mathrm{R}$ & $\mathrm{R}$ & $\mathrm{S}$ & $\mathrm{R}$ & 0 \\
6 & $\mathrm{R}$ & $\mathrm{R}$ & $\mathrm{R}$ & $\mathrm{S}$ & 0 \\
7 & $\mathrm{~S}$ & $\mathrm{R}$ & $\mathrm{R}$ & $\mathrm{S}$ & 0 \\
8 & $\mathrm{R}$ & $\mathrm{S}$ & $\mathrm{R}$ & $\mathrm{R}$ & 0 \\
9 & $\mathrm{~S}$ & $\mathrm{R}$ & $\mathrm{S}$ & $\mathrm{R}$ & 0 \\
10 & $\mathrm{R}$ & $\mathrm{S}$ & $\mathrm{R}$ & $\mathrm{S}$ & 1 \\
11 & $\mathrm{~S}$ & $\mathrm{~S}$ & $\mathrm{R}$ & $\mathrm{S}$ & 1 \\
& $\mathrm{~S}$ & $\mathrm{R}$ & $\mathrm{S}$ & $\mathrm{S}$ & 1 \\
12 & $\mathrm{Sredicted}$ patterns $(=47 / 50(94 \%))$ & & \\
13 & $\mathrm{~S}$ & $\mathrm{~S}$ & $\mathrm{~S}$ & $\mathrm{~S}$ & 17 \\
14 & $\mathrm{~S}$ & $\mathrm{~S}$ & $\mathrm{~S}$ & $\mathrm{R}$ & 16 \\
15 & $\mathrm{~S}$ & $\mathrm{~S}$ & $\mathrm{R}$ & $\mathrm{R}$ & 8 \\
16 & $\mathrm{~S}$ & $\mathrm{R}$ & $\mathrm{R}$ & $\mathrm{R}$ & 2 \\
\hline column & $\mathrm{R}$ & $\mathrm{R}$ & $\mathrm{R}$ & $\mathrm{R}$ & 4 \\
totals & 45 & 43 & 34 & 20 & \\
\hline
\end{tabular}

$68 \%$, and $40 \%)$ declines monotonically with the difference between $p$ and $\lambda p(.19, .15$, .10 , and .05). These differences in proportion across problems are significant (Cochran's $\left.Q=48.63>16.26_{(.001,3 d . f .)}\right)$. Moreover, using a model of quasi-independence, the hypothesis that the non-predicted patterns occur randomly cannot be rejected (Pearson's $\left.X^{2}=3.02<4.61_{(.10,2 \text { d.f. })}\right)$.

The format of questions 1 through 4 is virtually identical to the two-stage format employed by Kahneman and Tversky (1979). As such, according to the explanation for violations of the reduction-of-compound-lotteries axiom proposed by Loomes and Sugden (1982), agents should have interpreted each of choices 1 through 4 as involving statistically dependent actions. If so, however, regret theory would predict no switching between $\mathrm{S}$ and $\mathrm{R}$. The observed switching from $\mathrm{S}$ to $\mathrm{R}$ is also difficult to attribute to the subproportionality of prospect theory's decision-weighting function, since it applies only to "small" probabilities. Across all four problems, however, the smallest probabilities are .25 and .2, occurring in Problem 4. Moreover, even if these were sufficiently small as to produce a reversal between Problems 3 and 4, subproportionality would not imply reversals between Problem 2 (in which the probabilities in the terminal lotteries are .75 and 
.60 ) and Problem 3 (where the probabilities are .5 and .4). Nonetheless, there are significant decreases in the proportion of subjects choosing the S option between Problems 2 $(86 \%)$ and $3(68 \%)\left(X^{2}=5.33>5.02(.025\right.$, l.d.f. $)$, as well as between Problems $3(68 \%)$ and $4(40 \%)\left(X^{2}=8.89>7.88_{(.005}\right.$, Id.f. $\left.)\right)$. These results, in turn, suggest that the key to producing expected utility violations via manipulation of probability values lies not in the magnitude of the probabilities, as implied by prospect theory's decision-weighting function, but in the magnitude of the difference in probabilities consistent with $\sim p$ being an $\varepsilon$-difference similarity.

\section{Conclusions}

Evidence demonstrating choice anomalies has been widely interpreted as indicating that people do not wish to obey expected utility and has resulted in a plethora of alternative models of choice under uncertainty. This article has explored the alternative hypothesis that individuals who are unwilling or unable to rationally resolve choices between alternatives which are in some sense "close" in expected utility or value base their choices upon simple comparative procedures involving the equality/inequality or similarity/dissimilarity of prizes and probabilities across alternatives. As such, the model proposed here may be seen as a complement to, rather than a substitute for, expected utility, or, for that matter, expected value, maximization. The economic relevance of behavior implied by similarity judgments depends upon whether such judgments are employed, because relying upon a more coherent procedure is merely costly (in which case, incentives should eliminate departures from rationality) or prohibitively so (in which case, similarity judgments may influence choice in decisions of importance). Recent inquiries as to whether financial incentives matter for the types of behaviors discussed in this article (e.g., Camerer (1989), and Battalio, Kagel, and Jiranyakul (1990)) suggest the latter.

In any case, the ability of the model presented here to explain a large subset of choice anomalies and, in the process, to draw the descriptive validity of two very different alternatives to expected utility into question is significant. For representations of choices where for each of the $j$ pairs of comparisons made, $p_{1 j}$ is equal to $p_{2 j}$, the model predicts violations of equivalence, monotonicity, and transitivity heretofore unique to regret theory. For other representation or manipulations of choices, the model proposed implies behaviors which formed the basis for prospect theory. The appearance of "certainty effects," subproportionality, risk aversion for gains, and risk seeking for losses are examples. In addition, the model uniquely predicts violations of tenets of rationality, such as dominance among statistically independent prospects, the intransitive choice pattern reported by Tversky when probabilities appear similar, the opposite pattern when prizes appear similar, and systematic preference reversals between different two-stage representations of a choice between the same single-stage lotteries. All these predictions have, at least preliminary, experimental support. In sum, the results reported in this article suggest that a more extensive inquiriy into the role of similarity judgments in choice is in order. 


\title{
Acknowledgments
}

\author{
Robyn Dawes, John Dickhaut, Steven Klepper, and Patrick Sileo, and an anonymous \\ referee provided valuable comments on earlier versions of this article. Remaining errors \\ are the responsibility of the author.
}

\section{Notes}

1. The requirement that agents be indifferent between two options corresponding to the same probability distribution.

2. The requirement that agents prefer stochastically dominating alternatives.

3. That is, given choices between $\mathrm{A}:\{-\$ 3000, .02 ; \$ 0, .98\}$ and $\mathrm{B}:\{-\$ 6000, .01 ; \$ 0, .99\}$ and between $\mathrm{A}^{\prime}$ : $\{-\$ 3000, .90 ; \$ 0, .10\}$ and $B^{\prime}\{-\$ 6000, .45 ; \$ 0, .55\}$, agents tend to exhibit the response pattern $A B^{\prime}$.

4. For axiomatizations of such models, see Vincke (1980) and Nakamura (1988).

5. That is, for all $x_{g}$ and $x_{h} \in X: x_{g}>x_{x_{h}} \Rightarrow$ not $x_{h}>{ }^{x} x_{g}$, same for $p$ 's $\in[0,1]$, and for all $x_{f}>x_{g} x_{h} \in X: x_{f}$ $>x_{x_{g}}, x_{g}>x_{x_{h}} \Rightarrow x_{f}>x^{x}$, same for $p$ 's $\in[0.1]$.

6. That is, for all $x_{g}$ and $x_{h} \in X: x_{g}-x^{x} x_{h} \Rightarrow x_{h} \sim x^{x} x_{g}$, same for $p$ 's $\in[0,1]$.

7. More precisely, the predictions discussed will apply for prizes ordered "best," "good," "bad"; or "worst," "bad," "good."

8. Common consequence effects occur when replacing the prize value in a prize-probability pair common to two lotteries influences an agent's choice (e.g., S: $\{\$ 2400 ; 1 ; \$ 0, .0\}>\mathrm{R}:\{\$ 2400.66 ; \$ 2500, .33 ; \$ 0, .01\}$, but $\mathrm{R}^{\prime}:\{\$ 2500, .33 ; \$ 0, .67\}>\mathrm{S}^{\prime}:\{\$ 2400, .34 ; \$ 0, .66\}$ (the latter pair of lotteries being obtained from the former by replacing the common component $(\$ 2400, .66)$ in the first pair with the common component $(\$ 0, .66))$.

9. Experimental evidence supporting Predictions 1 and 2 is somewhat ambiguous. With state-dependent matrix representations similar to Rla and b, Loomes (1988) and Loomes and Sugden (1987) report results indicating an absence of common ratio effects in choices involving certain sets of prize values, but evidence of common ratio effects for other sets. Starmer and Sugden (1989) report an absence of common ratio violations for choices involving losses, but find violations for choices involving gains. Starmer and Sugden (1987) report an absence of common consequence effects for lotteries represented in state-dependent matrices similar to Rla and b, but note that Slovic and Tversky (1974) and Moskowitz (1974) did report common consequence violations in similar experiments. In contrast, evidence supporting Prediction 3 is unequivocal: Loomes and Sugden (1987), Loomes (1988), and Starmer and Sugden (1989) all report observing systematic juxtaposition effects consistent with Prediction 3.

10. In the shorthand employed to denote possible choice patterns across the lottery triple (e.g., ABC), the first letter indicates the choice made between $\mathrm{A}$ and $\mathrm{B}$, the next letter the choice made between $\mathrm{B}$ and $\mathrm{C}$, and the last letter the choice made between $\mathrm{A}$ and $\mathrm{C}$ (thus, $\mathrm{ABC}$ implies choosing $\mathrm{A}$ over $\mathrm{B}, \mathrm{B}$ over $\mathrm{C}$, and $\mathrm{C}$ over A).

11. Note that with Coffering $x_{3}$ with certainty, the intransitive choice pattern BCA is the pure choice analog to the preference reversals reported by Lichtenstein and Slovic (1971), Grether and Plott (1982), and others. Specifically, choosing B over A, corresponds to choosing the pbet over the \$bet, while choosing C over B and $A$ over $C$ correspond to assigning a lower price to the $p$ bet than to the $\$ b e t$.

12. Employing state-dependent matrix representations similar to RII for lotteries involving gains, Loomes, Starmer, and Sugden (1991) report systematic violations of the BCA variety, while Loomes and Taylor (1992) report systematic intransitivities of the opposite type for choices involving losses. Both results are consistent with Prediction 4.

13. Loomes and Sugden (1992) find that individuals do violate stochastic dominance in the manner consistent with Prediction 5. They did not find that violations of equivalence differed significantly from a $50 / 50$ split (indifference statements were not allowed), although this result is not inconsistent with Prediction 5, which only requires that the lottery $A^{\prime}$ not be chosen systematically.

14. Recall from footnote 10 that Loomes and Sugden (1992) found systematic violations of stochastic dominance, but not violations of equivalence required by regret theory, but only allowed by similarity. Indeed, 
Loomes and Sugden (1992) speculate, consistent with the hypothesis put forth in this article, that "this raises the possibility that the violations of monotonicity that we found ... were the result of some factor other than regret."

15. Specifically, for predictions regarding choices between $\mathrm{Sa}$ and $\mathrm{Ra}$, and between $\mathrm{Sb}$ and $\mathrm{Rb}$, and in choices between probabilistically equivalent lotteries.

16. Specifically, the $6 \%$ (red) and $1 \%$ (green) chances of winning $\$ 45$ in $A$ are combined to produce a $7 \%$ (red) chance of $\$ 45$ in $\mathrm{A}^{\prime}$, and the $1 \%$ (blue) and $2 \%$ (yellow) chances of losing $\$ 15$ in $\mathrm{B}$ are combined to produce a $3 \%$ (yellow) chance of losing $\$ 15$ in $\mathrm{B}^{\prime}$.

17. These results might also come about if agents whose preferences are as assumed in regret theory choose according to similarity judgments when incentives are low, rather than incur costs associated with transforming the alternatives into their appropriate matrix representation. Harless (1992), however, presents evidence revealing failures of regret theory which are difficult to attribute to it being costly to produce the appropriate matrix representation of the alternatives. Specifically, he examined regret theory's predictions regarding the occurrence of common ratio violations due to the juxtaposition of prizes as in Prediction 3. For standard matrix representations of the alternatives shown below on the left, with probabilities of states operationalized as lottery tickets numbered 1 through 100 , the predictions of regret theory held. However, when the alternatives were presented in the proportional format on the right, regret effects vanished.

1

60

\begin{tabular}{|r|r|l|}
\hline 8000 & 0 & 0 \\
\hline 0 & 0 & 20000 \\
\hline
\end{tabular}

$1 \quad 61$

$60 \quad 100$

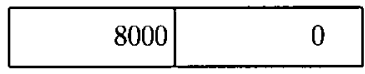

1 71 100

70

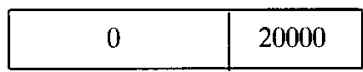

The only differences between the proportional and the matrix representations are that in the former the lotteries are separated by a horizontal space and the vertical lines separating identical outcomes have been removed. Harless (1992) notes that the absence of juxtaposition effects here is hard to attribute to insufficient incentives, as "discerning the juxtaposition of consequences requires just the tiniest bit more effort than required in the matrix format. .." (i.e., that associated with extending the vertical lines dividing prizes in lotteries up and down). Note also that, in the context of the Similarity model, the absence of regret effects in this format is not surprising, as, once the vertical lines are removed, probabilities in one alternative are no longer compared with probabilities of of identical value in the other alternative.

18. Within each questionnaire, choices between lotteries were presented on separate pages, with $A$ to the left of $B, B$ to the left of $C$, and $C$ to the right of $A$, or vice versa, and with questions sequenced in all possible orders (for a total of 12 questionnaire types).

19. The certainty effect was also hypothesized based upon the observation that people exhibited common ratio violations of independence when one of the outcomes was to be received with certainty (e.g., $\{\$ 3000\}>$ $\{\$ 4000, .8\}$ but $\{\$ 4000, .20\}>\{\$ 3000, .25\})$.

\section{References}

Allais, M. (1953). "Le Comportement de l'homme rationel devant le Risque, critique des postulates et axiomes de l'ecole americane," Econometrica 21, 503-546.

Battalio, R., J. Kagel, and R. Kiranyakul. (1990). "Testing Between Alternative Models of Choice Under Uncertainty: Some Initial Results," Joumal of Risk and Uncertainty 3(1), 25-50. 
Camerer, C. (1989). "An Experimental Test of Several Generalized Utility Theories," Joumal of Risk and Uncertainty 2(1), 61-104.

Friedman M, and L.. Savage. (1948). "The Utility Analysis of Choices Involving Risk," The Joumal of Political Economy 56, 279-304.

Grether, D., and C. Plott. (1979). "Economic Theory of Choice and the Preference Reversal Phenomenon," The American Economic Review 69, 623-638.

Harless, D. (1992). "Actions Versus Prospects: The Effects of Problem Representation on Regret," The American Economic Review 82, 634-650.

Kahneman, D., and A. Tversky. (1979). "Prospect Theory: An Analysis of Decisions Under Uncertainty," Econometrica 47, 263-291.

Leland, J. (1991) . "Choice Paradoxes as Decision Errors," Carnegie Mellon University Working Paper.

Leland, J. (1992). "Similarity, Intransitivities, and the Preference Reversal Phenomenon," Carnegie Mellon University Working Paper.

Lichtenstein, S., and P. Slovic. (1971). "Reversals of Preference Between Bids and Choices in Gambling Decisions," Joumal of Experimental Psychology 89, 46-55.

Loomes, G. (1988). "Further Evidence on the Impact of Regret and Disappointment in Choice Under Uncertainty," Economica 55, 47-62.

Loomes, G., C. Starmer, and R. Sugden. (1991). "Observing Violations of Transitivity by Experimental Methods," Econometrica 59, 425-439.

Loomes, G., C. Starmer, and R. Sugden. (1992). "Are Preferences Monotonic? Testing Some Predictions of Regret Theory," Economica 59, 17-33.

Loomes, G., and R. Sugden. (1982). "Regret Theory: An Alternative Theory of Rational Choice Under Uncertainty," The Economic Joumal 92, 805-824.

Loomes, G., and R. Sugden. (1987). "Testing for Regret and Disappointment in Choice Under Uncertainty," The Economic Joumal 97, 118-129.

Loomes, G., and C. Taylor. (1992). "Non-transitive Preferences over Gains and Losses," The Economic Joumal 102, 357-365.

Moskowitz, H. (1974). "Effects of Problem Representation and Feedback on Rational Behavior in Allais and Morlat-type Problems," Decisions Sciences 5, 225-242.

Kakamura, Y. (1988). "Expected Utility with an Interval Order," Joumal of Mathematical Psychology, 32, 298-312.

Rubinstein, A. (1988). "Similarity and Decision-making Under Risk (Is There a Utility Theory Resolution to the Allais Paradox?)," Joumal of Economic Theory 46, 145-153

Slovic, P., and A. Tversky. (1974). "Who Accepts the Savagc Axiom," Behavional Science 19, 368-373.

Starmer, C., and R. Sugden. (1987). "Experimental Evidence of the Impact of Regret on Choice Under Uncertainty," The Economics Research Center, Discussion Paper \#23.

Starmer, C., and R. Sugden. (1989). "Probability and Juxtaposition Effects: An Experimental Investigation of the Common Ratio Effect," Joumal of Risk and Uncertainty 2(2), 159-178.

Tversky, A. (1969). "Intransitivity of Preferences," Psychological Review 76, 31-48.

Tversky, A., and D. Kahneman (1986). "Rational Choice and the Framing of Decisions," Joumal of Business. S251-S278.

Vincke, P. (1980). “Linear Utility Functions on Semiordered Spaces,” Econometrica 48, 771-775. 
Copyright of Journal of Risk \& Uncertainty is the property of Kluwer Academic Publishing / Business and its content may not be copied or emailed to multiple sites or posted to a listserv without the copyright holder's express written permission. However, users may print, download, or email articles for individual use. 